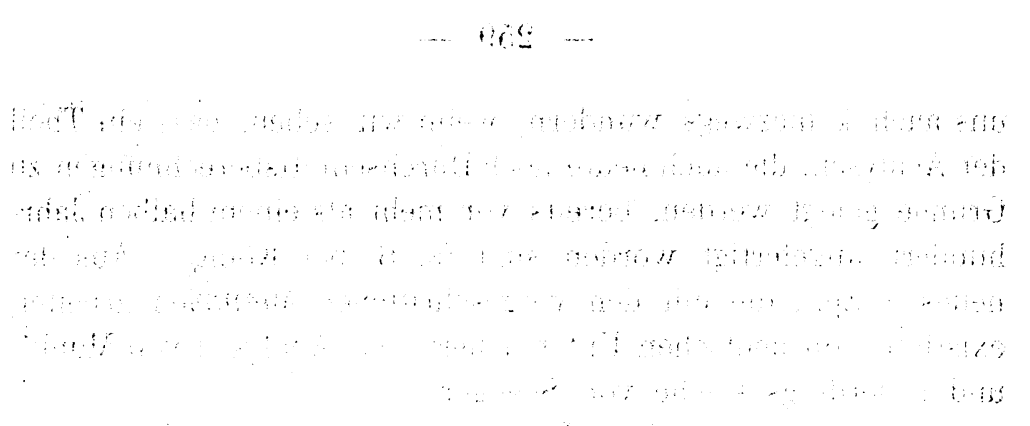

\title{
Ueber Eselsmilch.
}

\author{
von \\ Arthur Schlossmann.
}

(Aus dem Laboratorium für organische Chemie an der technischen Hochschule zu Dresden.) (Der Redaction zugegangen am 20. April 1397.)

$\therefore i \hat{i}$

In einer früheren Arbeit ${ }^{1}$ ) habe ich es als wünschenswerth bezeichnet, die Milch der Frau einerseits, die verschiedener Säugethiere andererseits vergleichend nach denselben Methoden zu untersuchen, um auf diese Weise physiologische und chemische Anhaltspunkte zu gewinnen, die eine wirklich rationelle Ernährung der aus irgend welchen Gründen der Mutterbrust entbehrenden Säuglinge ermöglichen. Es soll von diesem Gesichtspunkte ausgehend im Folgenden über die Zusammensetzung der Eselsmilch berichtet werden, zumal gerade die Milch dieses Thieres in Deutschland noch wenig eingehend untersucht worden ist und sich eine ganze Kette von unrichtigen Vorstellungen an einige veraltete uns aus Frankreich überkommene Analysen angeschlossen haben. Aus naheliegenden Gründen ist die Eselsmilch ein ziemlich rares Untersuchungsobject und die meisten Untersucher, die über diese Frage etwas veröffentlicht haben, stützen sich nur auf eine oder wenige Analysen. Die dabei erhaltenen Resultate sind sehr widersprechende und stimmen schlecht überein. Dies wird

1) Schlossmann, Ueber die Eiweissstoffe der Milch und die Methoden ihrer Trennung. Diese Zeitschrift, Bd. XXII, p. 197. 
uns auch keineswegs wundern, wenn wir sehen, dass ein Theil der Analysen, die auch heute noch Durchschnittsberechnungen zu Grunde gelegt werden, bereits vor mehr als einem halben Jahrhundert angefertigt worden sind (z. B. bei König). Aus der neuesten Zeit, die mit den vorgeschrittenen Methoden arbeitet, existiren von deutschen Untersuchern nur Analysen von Munk ${ }^{1}$ ) und neuerdings solche von Seeliger ${ }^{2}$ ).

Bereits diese beiden Untersucher widerlegen das immer kritiklos weiter verbreitete Märchen von der physiologischchemischen Gleichwerthigkeit der Frauen- und Eselsmilch. Nachdem ich bereits früher ${ }^{3}$ ) darauf hingewiesen hatte, dass die vorliegenden Analysen," soweit "solche eben neueren Datums sind, durchaus nicht dafür sprechen, dass die Eselsmilch ein geeigneter Ersatz der Frauenmilch sei, und ich für diese meine Ansicht auch schon 2 eigene Untersuchungen ins Feld führen konnte, habe ich nunmehr die in Dresden gebotene Gelegenheit, sich von der Unzulänglichkeit der Eselsmilch als Säuglingsnährung $\mathrm{zu}$ überzeugen, $\mathrm{zu}$ fortlaufenden Untersuchungen benutzt:

Es wurde zu diesem Zwecke vom 1. Februar bis zum 4. März incl. täglich der Fettgehalt und der Gesammtstickstoffgehalt der von der Eselsmilchgewinnungsgenossenschaft Hellerhof verkauften Milch festgestellt und zwar jedesmal an je 2 Proben. Die Fettbestimmungen geschahen nach der Methode von Gerber-Gärtner. (Fällung des Eiweisses durch concentrirte Schwefelsäure, Lösen des Fettes' in Amylalkohol, Centrifugiren, volumetrische Bestimmung der Fettschicht). Man bekommt auf diese Weise, wie ich mich durch wiederholte Kontrolle durch Gewichtsanalyse auch bei der Eselsmilch überzeugt habe, genügend genaue Werthe.

1) Munk und Uffelmann, Die Ernährung des gesunden und kranken Menschen. Wien u. Leipzig. 2. Aufl. 1891. S. 121.

2) Seeliger, veröffentlicht bei Klemm, Jahrbuch f. Kinderheilkunde, Bd. XLIII, p. 381.

3) Verhandlungen der Ges. für Natur- u. Heilkunde in Dresden. Sitzungen vom 2. Mai und vom 9. Mai 1896. 
Täglicher Fett- und Stickstoffgehalt der Eselsnilch

vom 1. Februar - 4. Mărz 1897.

\begin{tabular}{|c|c|c|c|c|c|}
\hline Datum & Fettgehalt & N-Gehalt & Datum & Fettgehalt & N-Gehalt \\
\hline & Procent. & Procent. & & Procent & Procent \\
\hline 1. Febr. & 0,20 & 0,2604 & 17. Febr. & 0,40 & $0,2: 324$ \\
\hline 2. , & 0,30 & 0,2548 & 18. , & 0,40 & 0,2338 \\
\hline 3. , & 0,30 & 0,2632 & 19. , & 0,30 & 0,2310 \\
\hline 4. , & 0,20 & 0,2688 & 20. & 0,20 & 0,2268 \\
\hline 5. : & 0,30 & 0,2604 & 21. , & 0,40 & 0,2268 \\
\hline 6. " & 0,40 & 0,2604 & 22. " & 0,15 & 0,2170 \\
\hline 7. . & 0,60 & 0,2520 & 23. & 0,40 & 0,2198 \\
\hline 8. ", & 0,60 & 0,2604 & 24. & 0,30 & 0,2478 \\
\hline 9. , , & 0,30 & 0,2660 & 25. & 0,50 & 0,2464 \\
\hline 10. , & 0,40 & 0,2590 & 26. & 0,60 & 0,2534 \\
\hline 11. , & 0,30 & 0,2590 & 27. : & 0,30 & 0,2576 \\
\hline 12. ", & 0,50 & 0,2534 & 28. , & 0,30 & 0,2576 \\
\hline 13. , & 0,40 & 0,2268 & 1.März & 0,40 & 0,2436 \\
\hline 14. ", & 0,40 & 0,2422 & 2. , , & 0,20 & 0,2464 \\
\hline 15. ", & 0,30 & 0,2366 & 3. , & 0,60 & 0,2352 \\
\hline 16. " & 0,30 & 0,2254 & 4. ,, & 0,40 & 0,2562 \\
\hline
\end{tabular}

Die Stickstoffbestimmungen geschahen in gewointer Weise nach Kjeldahl. Die obenstehende Tabelle gibt übır die täglichen Befunde Aufschluss. Ebenfalls täglich wurce das spec. Gewicht und die Reaction festgestellt. Asche, Zucier und die Fractionirung der einzelnen Stickstoffträger kınnte nicht täglich durchgeführt werden, jedoch bezieht sich jede meiner folgenden Angaben auf den Durchschnitt von mindestens 6 Analysen.

Nach meinen Erfahrungen characterisire icl die Eselsmilch folgendermaassen:

Aussehen: Die Eselsmilch sieht weiss mit sinem Stich ins Bläuliche aus.

Geschmack: Fad-süsslich, wie stark gewässerte Kuhmilch.

Mikroskopisches Verhalten: Deckt wenişer als entsprechend verdünnte und entfettete Kuh- und Frauenmilch. Fettkügelchen spärlich, klein. 
Reaction: Ein Cubikcentimeter erfordert, um mit Phenolphtaleïn neutrale Reaction zu geben, $0,06 \mathrm{ccm}$. 1/10 Norm. Natronlauge mit Dimethylorange resp. Lakmustinctur 0,404 ccm. 1/10 Norm. Schwefelsäure. Es dürfte sich empfehlen, bei Aciditätsbestimmungen der Milch stets das Verhalten derselben gegenüber Phenolphtaleïn einerseits, Dimethylorange resp. Lakmustinctur andrerseits festzustellen. Aus der Differenz, die sich bei diesen beiden Bestimmungsarten ergibt, kann man Schlüsse auf die Gegenwart einer grösseren oder geringeren Menge gewisser Körper ziehen, die zwar Lakmus resp. Dimethylorange gegenüber deutlich alkalisch reagiren, jedoch einen Alkalizusatz erfordern, um gegenüber Phenolphtaleïn das übliche Verhalten alkalischer Lösungen zu zeigen. (Anorganische und organische Phosphorverbindungen?)

Specifisches Gewicht: 1033 (1031-1036).

Trockensubstanz: $11,15 \%$.

Asche: $0,399 \%$.

Zucker: $4,94 \%$.

Gesammtstickst off: $0,2431 \%$.

In maximo: $0,2688 \%$, in minimo: $0,2170 \%$.

Von dem Gesammtstickstoff des Eiweisses waren als Caseïn im Durchschnitt zu betrachten $3 / 4,1 / 4$ dagegen als Albumin.

Als Eiweiss fällbar mit denjenigen Fällungsmitteln, die alles Eiweiss, aber 'auch nur dieses, fällen (Trichloressigsäure und Salicylsulfonsäure), erwiesen sich etwa $86 \%$ des Gesammt-N-Gehaltes.

Phosphorfleischsäure (vgl. Siegfried, diese Zeitschrift, Bd. XXII, p. 575) $0,1205 \%$.

Fettgehalt: $0,364 \%$.

In maximo $0,6 \%$, in minimo $0,15 \%$.

Die täglichen Schwankungen in Fett- und Eiweissgehalt sind, wenn man berücksichtigt, dass es sich wohl um eine Mischmilch aus einer nicht zu zahlreichen Herde gehandelt hat, keine allzu beträchtlichen. Das Mehr oder Weniger an Gesammt-N ist übrigens speciell an einen vermehrten oder geringeren Gehalt desjenigen Theiles $\mathrm{N}$ gebunden, der nicht dem Caseïn entstammt, da die Menge des letzteren ziemlich constant bleibt. 
-196 Es, sergibt: sich also folgendes Resultat der Analyse; dem ich vergleichsweise die Seeliger'schen und Munk'schen Zahlen gègenüberstelle:

\begin{tabular}{|c|c|c|c|c|}
\hline \multirow{2}{*}{ 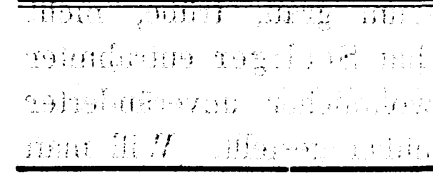 } & \multicolumn{4}{|c|}{ In 100 Theilen Milch finden : } \\
\hline & $\begin{array}{l}\text { Schloss- } \\
\text { mann }\end{array}$ & & II & \\
\hline Tröckensubstanz & 11,15 & - & - & $\therefore-1$ \\
\hline Gesammt-N & 0,243 & - & $一$ & - \\
\hline Als Eisweiss fällbarer $\mathrm{N}$ & 0,209 & 一 & 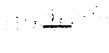 & $\because-1$ \\
\hline Cáseïn ${ }^{1}$ ) (incl. Spuren Globulin) & 0,981 & 1,01 & 0,654 & 0,7 \\
\hline Albumin 1) . . . . . . & 0,327 & $0 ; 37$ & 0,245 & 1,60 \\
\hline Phosphorfleischsäure . . . • & 0,120 & $*$ ) & *) & -1 \\
\hline Fett $\cdot \bullet \cdot \bullet \cdot$ & 0,364 & 0,45 & 0,94 & 1,6 \\
\hline Zucker • • . . • • : & 4,94 & 6,61 & 4,85 & 6,0 \\
\hline sche. . . . . . . . & 0,309 & 0,42 & 0,42 & 0,5 \\
\hline
\end{tabular}

Im physiologischen Verhalten gegenüber $1 / 10 \%$ iger $\mathrm{HCl}$ Lösung sowie verschiedenartigen Lablösungen habe ich einen Unterschied zwischen Eselsmilch und entsprechend präparirter Kuhmilch nicht gefunden: beide Proben gerannen jeweils gleich rasch und in genau der gleichen Art, ohne dass es möglich gewesen wäre, von grösseren oder feineren Flocken in dem einen oder anderen Falle zu sprechen. Dieser meiner Beobachtung scheint auf den ersten Blick eine Angabe Seeliger's zu widersprechen; dieser schreibt, dass entrahmte Kuhmilch, mit Magenextract behandelt, einen zusammenhängenden festen Kuchen, entrahmte Eselsmilch einen ganz feinflockigen Niederschlag gibt; weiter, dass Kuhmilch auf Pepsin- und Salzsäurezusatz hin nach einiger Zeit Gerinnung zeigt unter allmählicher Bildung eines festen Kuchens mit 2 Schichten: einer oberen

*) Statt dieser findet sich bei Seeliger ein Posten Lactoproteïn 0,09 resp. 0,072 .

1) Berechnet durch Multiplication des gefundenen N-Werthes mit 6,25. 
Fett- und unteren Käseschicht, Serumi getblich, i fast klar, afladenziehend,'Eselsmilch dagegen sofortige Abseheidung zarter Flocken, die sich absetzen. Zur Bildung eines zusammenhängenden Käsekuchens kam es hier nie, Serum grau, trube, nicht fadenziehend. Wie also ersichtlich, hat Seeliger entrahmter Eselsmilch entrahmte Kuhmileh, gewöhnlicher unveränderter Eselsmilch ebènsolche Kuhmilch gegenüber gestellt. Will man aber für die Säuglingsernährung geeignete Schlüsse ziehen oder aber das specielle Verhalten des Caseines zweier Milcharten vergleichen, so muss man selbstverständlich den Caseîngekalt in beiden Proben ebenso wie den Fettgehalt gleichstellen und auch die Unterschiede in Bezug auf das gelöste Albumin möglichst ausgleichen, da ja auch letzteres, wie ich $^{1}$ ) zeigen konnte, die Gerinnungsart wesentlich modificirt. Nimmt man also Lösungen, die gleiche Mengen Caseïn, Fett und Albumin enthalten, so zeigt sich in der Gerinnung zwischen Eselsmilch und Kuhmilch kein Unterschied mehr und alle weiteren auf Verdauungsversuchen, die diesen Anforderungen nicht gerecht werden, aufgebauten Folgerungen sind Trugschlüsse.

Betrachten wir nochmals die Resultate meiner Untersuchungen, so ergibt sich ohne Weiteres, dass von einer besonderen Aehnlichkeit zwischen dem physiologisch-chemischen Verhalten der Frauen- und Eselsmilch keine Rede ist. Der Hauptunterschied zwischen Frauenmilch und der Milch aller anderen Thiere besteht in dem Verhältniss zwischen $\mathrm{N}$-haltiger Substanz und Fett respp. N-freier Substanz. Während in der Frauenmilch auf:

kommen:

$1 \mathrm{gr}$ Fett $0,34 \mathrm{gr}$ N-haltige Substanz kommen,

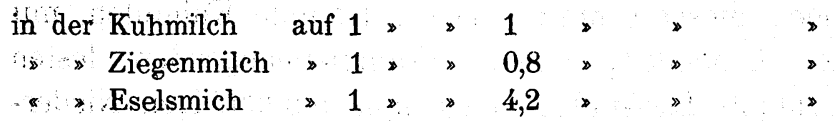

Wollte also ein Säugling im 3. Monat die erfahrungsgemäss von ihm benöthigte Menge von $34,96 \mathrm{gr}$ Fett (in $920 \mathrm{gr}$

1) Schlossmann, Ueber Art, Menge und Bedeutung der stickstoffhaltigen Substanzen in der Frauenmilch. Verh. der Ges. für Kinderheilkunde 1896, p. 75, und Allgem. Med. Centralzeit. 1896. 
Muttermilch) aus Eselsmilch consumiren, so müsste er zugleich 146,8 gr N-haltige Substanz aufnehmen, während das Brustkind nur 11,96 gr auf die gleiche Menge Fett erhält. Das mit Eselsmilch genährte Kind müsste zudem ca. 10 Liter Eselsmilch trinken, um seinen täglichen Fettbedarf zu decken. Gibt man einem Kinde dieses Alters jedoch, wie Klemm ${ }^{1}$ ) dies empfiehlt, nur 900 gr Eselsmilch pro die, so erhält es 3,33 gr Fett oder 31,63 gr weniger, als es bedarf. Dieses Manco an Fett entspricht einem physiologischen Nutzeffect von 294 Calorien, die dem Kinde täglich entzogen werden. In 920 gr Muttermilch erhält jedoch das Brustkind Nahrungsstoffe, die etwa 608 Calorien entsprechen; fast die Hälfte davon würde einem nach Klemm mit Eselsmilch genährten Kinde abgehen. Wie sich daher der Lehrsatz: "Die Eselsmilch genügt wenigstens bis zum Beginn des vierten Monats als ausschliessliche Nahrung des Säuglings» mit den elementarsten Sätzen der Physiologie verträgt, dürfte jedem, der gewohnt ist, derartige Fragen wissenschaftlich zu erörtern, unverständlich sein.

Wenn ich auf diese Momente hier überhaupt näher eingegangen bin, so geschah dies nur aus dem Grund, um darauf hinzuweisen, dass wir in der Eselsmilch durchaus keinen passenden Ersatz der Muttermilch erblicken dürfen ${ }^{2}$ ); das lehrt ja schon ein einfacher Blick auf Munk's, Seeliger's und meine Analysen. Es ist daher zu hoffen, dass die immer und immer wieder behauptete Gleichwerthigkeit der Eselsmilch mit der Milch der Frau, eine Ansicht, die sich übrigens auch in wissenschaftlichen Werken vertreten findet und sich neuerdings in Dresden sogar in der Tagespresse aufdringlich breit macht, endgültig zu den widerlegten Irrthümern gezählt werden wird.

1) Jahrbuch für Kinderheilkunde $B$. XLIII.

2) Eine genaue Litteraturübersicht über die Frage der Ernährung mit Eselsmilch wird sich in einer demnächst im Archiv für Kinderheilkunde zur Veröffentlichung gelangenden Arbeit: «Die künstliche Säuglingsernährung » finden. 